\title{
PERSPECTIVE \\ Industry Support of Medical Research: Important Opportunity or Treacherous Pitfall?
}

\author{
William M. Tierney, $M D^{1,2}$, Eric M. Meslin, $P h D^{2,3}$, and Kurt Kroenke, $M D^{1,2,4}$ \\ ${ }^{1}$ Regenstrief Institute, Inc. Indianapolis, IN, USA; ${ }^{2}$ Indiana University School of Medicine, Indianapolis, IN, USA; ${ }^{3}$ Indiana University Center for Bioethics, \\ Indianapolis, IN, USA; ${ }^{4}$ Roudebush VA Center for Health Information and Communication, Indianapolis, IN, USA.
}

Pharmaceutical and device manufacturers fund more than half of the medical research in the U.S. Research funding by for-profit companies has increased over the past 20 years, while federal funding has declined. Research funding from for-profit medical companies is seen as tainted by many academicians because of potential biases and prior misbehavior by both investigators and companies. Yet $\mathrm{NIH}$ is encouraging partnerships between the public and private sectors to enhance scientific discovery. There are instances, such as methods for improving drug adherence and post-marketing drug surveillance, where the interests of academician researchers and industry could be aligned. We provide examples of ethically performed industry-funded research and a set of principles and benchmarks for ethically credible academic-industry partnerships that could allow academic researchers, forprofit companies, and the public to benefit.

KEY WORDS: ethics, research; conflict of interest; public-private partnerships; research support.

J Gen Intern Med 31(2):228-33

DOI: $10.1007 / \mathrm{s} 11606-015-3495-\mathrm{z}$

(c) Society of General Internal Medicine 2015

F ederal funding of research has decreased over the past decade. ${ }^{1}$ At the same time, NIH has called for more collaboration between industry and academic investigators. For example, NIH's Discovering New Therapeutic Uses for Existing Molecules initiative will test more than 20 compounds from industry partners for their effectiveness against a variety of diseases and conditions. ${ }^{2}$ Accelerating Medicine Partnerships is a collaboration among NIH, ten pharmaceutical companies, and non-profit patient advocacy organizations to identify and validate the most promising biological targets of disease for new diagnostic and drug development. ${ }^{3}$ "Clearly, we need to speed the pace at which we are turning discoveries into better health outcomes," said NIH Director Collins. "NIH looks forward to working with our partners in industry and academia to tackle an urgent need that is beyond the scope of any one organization or sector". 4 Additionally, since passage of the Patent and Trademark Law Amendments

Received July 2, 2015

Revised July 16, 2015

Accepted July 23, 2015

Published online August 26, 2015
("Bayh-Dole") Act of $1980,{ }^{5}$ many academic institutions encourage faculty to patent and commercialize their discoveries, leading to mutually - scientifically and financially - beneficial partnerships between universities, their individual scientists, and private sector companies. In the wake of this engagement between academia and industry, and the enhanced scrutiny of industry payments to physicians prompted by passage of the Physician Payment Sunshine Act, ${ }^{6}$ universities nationwide are revising their guidelines for conducting research and managing conflicts of interest.

Industry and government together have consistently funded most medical research in the U.S. (Fig. 1). Notably, industry dominates: research funding by industry in 2012 was $\$ 68$ billion compared to $\$ 38$ billion from federal agencies. Moreover, between 1994 and 2012, industry funding of medical research grew by $147 \%$, compared with $48 \%$ for federal agencies, which was less than the $57 \%$ inflation during those years. ${ }^{7}$ The goal of federal research funding is to generate new knowledge that will enhance health and health care. The goal of research funding by for-profit companies is maximizing income to their shareholders. Increased knowledge and enhanced care, if they happen at all, are byproducts of the profit motive.

Can academicians' interest and industry's needs be aligned? For example, a company developing a new drug that may have fewer side effects might be interested in funding research into the incidence of adverse effects from currently marketed drugs. An academic researcher might have a strong interest in elucidating the adverse effect profile of that class of drugs when used in everyday settings among patients who are usually, if not always, excluded from pre-marketing studies. ${ }^{8}$ Studies of how drugs and devices are used in everyday practice and the outcomes of treatment should be of mutual interest and benefit to both academic researchers and industry. For example, Bristol-Myers Squibb was about to launch a new antipsychotic drug and contracted with one of us (WMT) to conduct a study of the incidence of weight gain and diabetes among patients taking any of the currently available antipsychotics; the results were published in JGIM. ${ }^{9}$

Both academia and industry have interests in postmarketing drug surveillance. ${ }^{10}$ The FDA requires companies to conduct post-marketing surveillance (phase IV studies) of new drugs and certain devices. Whereas academic 


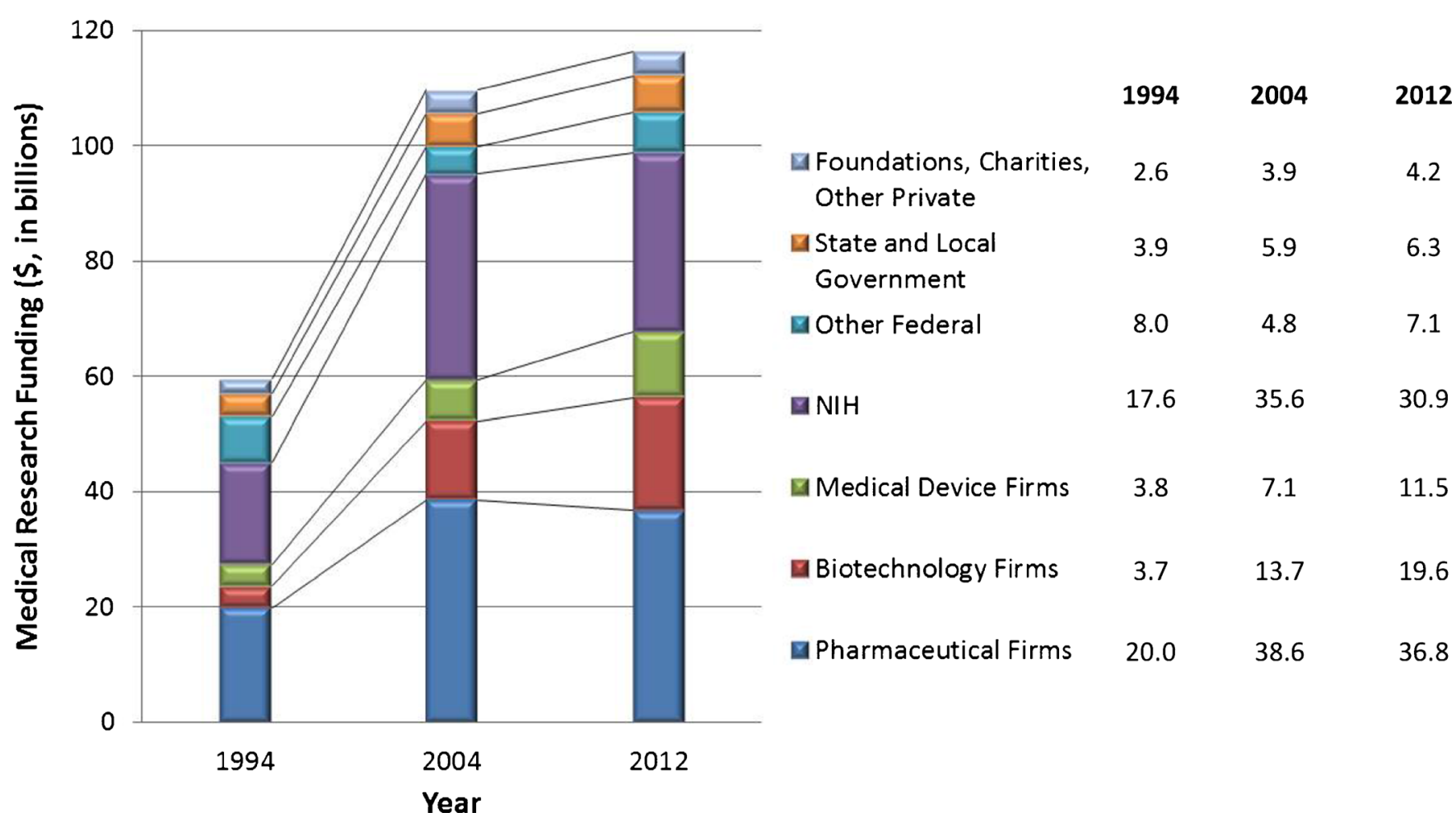

Fig. 1 Growth in medical research funding by source from 1994 to 2012 (\$, in billions) ${ }^{1}$

investigators are interested in true estimates of benefits and risks of new treatments, for-profit drug and device companies would want to report great benefits and low risks. We believe that academic researchers are more likely to perform unbiased post-marketing studies than either researchers employed by the company marketing the drug in question or for-profit research companies whose livelihood depends on satisfying their customers.

Academic researchers and industry scientists can also share interests in generating knowledge relevant to patient care. For example, clinicians hope and expect patients to take the medications they prescribe, and pharmaceutical companies benefit when patients take them. Thus both clinician-investigators and pharmaceutical companies have obvious interests in drug adherence and in developing and validating methods for assessing and improving adherence. Industry and academic investigators can also have mutual interests in improving our ability to identify, assess, and manage important patient outcomes. For example, the Regenstrief Institute has a five-year contract with Merck Sharp \& Dohme to develop and conduct mutually interesting and beneficial research projects. ${ }^{11}$ Researchers from the Regenstrief Institute and Indiana University and scientists from Merck propose collaborative one-year projects. A review committee comprising three senior investigators from both IU/Regenstrief and Merck reviews the proposals, eliminates some, and ranks the rest. Merck decides on its allocation to the collaboration each year, and then the review committee begins at the top of the rank list and funds projects until all allocated funds are expended.
Publication of study results in peer-reviewed journals is a required deliverable of each project. Table 1 shows projects funded in the first four years of this collaboration. Importantly, like federally funded projects, the grants reimburse the salaries of IU/Regenstrief investigators and professional staff. No bonuses or extra payments are made.

Industry-funded research has a risk of bias and misconduct that can mislead readers, ${ }^{12-14}$ consequently causing pain, suffering, and sometimes death. ${ }^{15}$ Neither academia nor the private sector is immune from ethical scrutiny or responsibility, though public perception rarely gives high marks to the pharmaceutical industry's ethical behavior. $^{16}$ The key is minimizing bias through rigorous studies devised, conducted, and reported by academic investigators whose income is not tied to the drug being evaluated. Each of these-research methods, how they are applied, and how results are reported-is a source of bias, regardless of funding source, that rigor and vigilance can minimize in order to generate new knowledge and patient benefit.

For example, Kroenke and his colleagues received funding from Pfizer to develop screening instruments for depression (the PHQ-9) and anxiety (the GAD-7). ${ }^{17}$ Both have become standard screening tools. The JGIM original article validating the PHQ- $9^{18}$ has been cited more than 3500 times, according to Web of Science ${ }^{19}$; it is JGIM's most highly cited article ever. Whereas both the PHQ-9 and GAD-7 are open-source and free to use, some survey instruments developed with federal funding require license fees, ${ }^{20}$ an unfortunate trend where 
Table 1 Funding of Projects in the First Four Years of the Regenstrief-Merck Collaboration

Project title

Project 1: Leveraging Regenstrief's electronic medical record (EMR) an capabilities to enhance subject recruiting

Project 2: Regenstrief-Merck Scholar's Award in Pharmacoepidemiology and Informatics

Project 3: Building a phenotype library using Regenstrief's EMR

Project 4a: Medication adherence in type 2 diabetes

Project 4b: Medication adherence in respiratory disorders

Project 5: Computerized reminders to promote medication adherence and utilization

Project 6a: Usage, Benefits, and Adverse Effects of Loop Diuretics in Patients with Heart Failure or Hypertension

Project 6b: Longitudinal Modeling of Heart Failure Progression

Project 7: Sensitivity analysis of Mini-Sentinel's protocol of active surveillance of acute myocardial infarction in association with antidiabetic agents

\section{Project 8: OpenMRS-Merck Strategic Collaboration}

Project 9: Calibrating evidence of drug risk by estimating clinical database bias

Project 10: Predictive modeling of drug-outcome associations

Project 11: Chronic kidney disease and resistant hypertension

Project 12: Collecting and incorporating patient-reported data into a medication adherence decision support system

Project 13: Development and feasibility of a medication adherence protocol for older adults with mild cognitive impairment Project 14: Investigation of physician reminders and recommendation scripts for HPV vaccination

Project 15: EMR-based detection and display of hypoglycemic risk in diabetic patients

Project 16: Melanoma algorithm development and validation

Project 17: Medication adherence in order adults with cognitive impairment (continuation of Project 13)

Osteoporosis Center of Excellence (OCOE)-1: Sub-Optimal Outcomes of Bisphosphonates Treatment in the Real World

OCOE-2: Renal Impairment in Osteoporosis

OCOE-3: Finding fractures and other phenotypes of high interest using EMR data and NLP

OCOE-4: Broadening osteoporosis-related data in the INPC

\section{Brief description of project goals}

Test the ability of a new identification system for EMR-enabled identification and recruitment of patients into clinical trials.

Create two annual visiting scholar positions whose focus will be primarily on the link between pharmacoepidemiology and biomedical informatics, leveraging big data analytics to: (1) improve patient care, (2) obtain better patient outcomes, and (3) lower costs.

Define and validate a set of three algorithms for defining phenotypes of interest, validating them against human interpretation of medical charts. Determine the patterns of use for medications prescribed to patients with type 2 diabetes mellitus, targeting medications for diabetes and associated metabolic disorders. Determine what patient-centered interview data might be collected, evaluate the merits of electronic monitoring of medications, and plan an intervention to improve adherence to medications. (This project was terminated due to feasibility issues.)

Test whether monitoring asthma medication adherence using prescription records and providing feedback to patients can improve drug adherence and asthma control among patients non-adherent to their inhaled controller medications.

Support more consistent and effective use of prescribed medications by identifying optimal physician and patient-directed strategies to improve appropriate medication adherence and utilization.

Identify and describe the characteristics of patients with diagnosed hypertension and heart failure using EMR data, and use prescription records to assess adherence for heart failure and hypertension medications, and relate clinical outcomes to medications prescribed and adherence. Utilize longitudinal EMR data to characterize the changes in ejection fraction and/or New York Heart Association chronic heart failure classification. Examine the impact of patient-specific covariates (drug treatment, intensity, age, weight, sex, etc.) on the rate of heart failure progression.

Better understand the sensitivity of risk estimates with respect to a set of parameters associated with design decisions. To accomplish this, the project evaluated a protocol from the Mini-Sentinel for the Active Surveillance of Acute Myocardial Infarction in Association with Use of Anti-Diabetic Agents.

Integrate a Merck business analyst and developer into the OpenMRS community who can comprehend and assist with open-source EMR development.

Develop methods to adjust results for more accurate answers to drug outcome research questions within the Indiana Network for Patient Care (INPC). Develop "database fingerprinting" methods that can be applied to any database in the Observational Medical Outcomes Partnership (OMOP) common data model format.

Develop and compare optimal predictive modeling techniques for identifying patients at risk of known drug outcomes.

Define the rates of resistant hypertension in populations with and without chronic kidney disease.

Determine whether non-interruptive claims-based adherence alerts enhanced with patient-reported data and tailored recommendations can increase the number of conversations clinicians and patients have about their adherence to oral medications for diabetes. (Due to delays encountered during development and deployment, this project was discontinued.)

Identify and quantify barriers to medication adherence in older adults with mild cognitive impairment.

Evaluate the effect of automated physician-targeted reminders and recommended scripts on first dose uptake of HPV vaccine and rates of return for second dose.

Develop a predictive model of hypoglycemia risk in patients taking insulin or sulfonylureas. Design an alert that will be delivered to providers accessing high-risk patients' EMRs.

Determine the sensitivity and positive predictive value of defining melanoma in the INPC database using EMR data and data derived from natural language processing (NLP).

Identify and quantify barriers to adherence in older adults with mild cognitive impairment.

Examine the prevalence and healthcare burden of osteoporosis patients who sustain fractures, lose bone mineral density, or remain osteoporotic despite being adherent to bisphosphonates treatment.

Quantify the unmet medical need in the area of comorbid osteoporosis and chronic kidney disease, as current osteoporosis therapies are not recommended in patients with moderate to severe chronic kidney disease. Develop and validate coding algorithms for fractures and other phenotypes to enhance observational studies.

Enhance researchers' ability to use the INPC for osteoporosis-related studies by creating the nation's largest repository of structured bone mineral density scans linked to EMR data. 
Table 1. (continued)

\begin{tabular}{|c|c|}
\hline Project title & Brief description of project goals \\
\hline OCOE-5: High-volume osteoporosis and patient access registry project & $\begin{array}{l}\text { Establish a large consenting cohort of patients (with links to their EMRs) } \\
\text { for rapid recruitment for future osteoporosis-related studies. }\end{array}$ \\
\hline OCOE-6: Disparities in osteoporosis treatment & $\begin{array}{l}\text { Use patient and provider characteristics in multivariate models to predict } \\
\text { which patients with osteoporosis, low bone mineral density, or fractures } \\
\text { receive treatment with bisphosphonates or other osteoporosis drugs. }\end{array}$ \\
\hline OCOE-7: Improving the capture, interpretation and use of DXA data & $\begin{array}{l}\text { Upload DXA data from selected health systems' radiology departments } \\
\text { and local clinics into INPC and assess the variability of longitudinal bone } \\
\text { mineral density measurements in the clinical setting. }\end{array}$ \\
\hline $\begin{array}{l}\text { OCOE-8: Diagnosis of atypical subtrochanteric fractures in the clinical } \\
\text { setting }\end{array}$ & $\begin{array}{l}\text { Estimate the proportion of atypical femur shaft fractures in women with } \\
\text { non-traumatic femur fractures, and identify clinical factors predicting } \\
\text { atypical femur shaft fractures. }\end{array}$ \\
\hline $\begin{array}{l}\text { Cross-Collaboration Initiative }(\mathrm{CCI})-1 \text { : Regenstrief-Merck Scholar's } \\
\text { Award in Pharmacoepidemiology and Informatics }\end{array}$ & $\begin{array}{l}\text { Create the first-ever combined pharmacoepidemiology-medical informatics } \\
\text { fellowship to develop and train world-class leaders at the intersection of } \\
\text { big data, pharmaceutical research, and health information technology. }\end{array}$ \\
\hline CCI-2: Regenstrief Boot Camp & $\begin{array}{l}\text { Hold an intensive two-day training seminar that will provide Merck and } \\
\text { local non-Regenstrief investigators knowledge of the wide-ranging } \\
\text { resources and capabilities available at the Regenstrief Institute. }\end{array}$ \\
\hline CCI-3: EMR Summit & $\begin{array}{l}\text { Hold a summit of commercial EHR and health IT developers to promote } \\
\text { awareness and adoption of innovations in evidence-based care, patient } \\
\text { safety, and user experience design. }\end{array}$ \\
\hline CCI-4: Natural Language Processing Core & $\begin{array}{l}\text { Expand Regenstrief's and Merck's capabilities to glean information from } \\
\text { text data in support of current and future projects. }\end{array}$ \\
\hline CCI-t: Electronic patient reported outcomes (ePRO) capture platform & $\begin{array}{l}\text { Create a flexible, scalable, and generalizable electronic platform for } \\
\text { generating and storing patient-reported outcomes on an unlimited variety } \\
\text { of topics. }\end{array}$ \\
\hline
\end{tabular}

Total Projects $=32$

Manuscripts: 14 submitted, 5 published or accepted for publication to date

29 Presentations at scientific meetings and conferences to date

patient-reported measures are frequently proprietary rather than in the public domain. ${ }^{21}$

Certainly there are well-documented cases where industryfunded research has been biased. For example, two systematic reviews found that studies sponsored by industry reported significantly greater benefits and less harm than studies with other sources of funding. ${ }^{22,23}$ Similarly, there are well-known examples where industry has squelched (or attempted to squelch) study results that were unfavorable to their products. $^{24,25}$ Pharmaceutical companies have also paid ghostwriters to draft reviews of drug treatment favorable to their products, and then sought academicians to "author" the articles, with the goal of biasing the medical literature. ${ }^{26}$ But does such obviously unethical behavior by some investigators and companies mean that industry-funded research can never be conducted by academic scientists without the results being questioned? We argue that academic-industry relationships can be "ethically credible," meaning that specific ethical principles are followed that minimize the risk that industry funding will bias the planning, conduct, or reporting of studies. Indeed, academic-industry relationships are not only possible, they are desirable as a means to maximize discoveries and patient benefits as federal research dollars are dwindling.

An example of an ethically credible partnership was the ARTIST study that was funded by Eli Lilly to assess the effects of different selective serotonin reuptake inhibitors (SSRIs) on depression and other outcomes in primary care. ${ }^{27}$ The sponsor had postulated that its SSRI (fluoxetine) would be more effective than two competing SSRIs (sertraline and paroxetine). However, the study found no differences among the three
SSRIs as reported in a high-impact journal (JAMA), despite not favoring the sponsor's drug. Indeed, the evidence supporting "funding bias" has recently been questioned by social scientists as well as the Cochrane collaboration. ${ }^{28,29}$

To counter potential bias due to industry funding of research, the Regenstrief Institute commissioned one of us (EMM) and his colleagues at the Indiana University Center for Bioethics (IUCB) to review Regenstrief's collaboration with Merck. ${ }^{11}$ Reviews of this kind are rare, but have been reported elsewhere. ${ }^{30}$ During the second year of the five-year collaboration, IUCB faculty and staff reviewed the contract between Regenstrief and Merck, assessed the bioethics literature concerning industry-funded research, surveyed Indiana University/Regenstrief investigators and staff engaged in one or more Merck-funded activities, and developed a set of principles and benchmarks for ethically credible academicindustry partnerships (Table 2). IUCB reviewers found the Regenstrief-Merck collaboration to be ethically conducted overall, but that it could be improved, especially in communicating the collaboration's policies and operating principles to all faculty and staff participants. ${ }^{31}$ The policies and procedures governing the Regenstrief-Merck collaboration were deemed to address the key ethical issues. Several benchmarks were not fully met, and the report made specific recommendations that the collaboration's leaders followed. In subsequent years, the collaboration has met all benchmarks. Specific recommendations followed were to 1) increase transparency and enhance trust by more fully educating all investigators and staff on the collaboration's policies and procedures; 2) broadcast the processes for ranking projects and selecting those to be funded; 3 ) 
Table 2 Principles and Benchmarks for Ethically Credible Academic-Industry Partnerships

\begin{tabular}{l}
\hline Principle \\
\hline Academic freedom \\
Conflict of interest policy
\end{tabular}

and management

Intellectual property

Data-sharing, access

Effective governance

Protection of human subjects

Publication

Social, scientific and industrial value

Transparency

\section{Benchmark}

1. Promote investigator-initiated science and protect the ability to attract and maintain federal research support 2. Permit investigators to initiate or continue collaboration with any other qualified group, person or entity 3 . Ensure that all investigators involved in the partnership are given equal opportunity to submit proposals for funding

4. Avoid obligating faculty to work outside their own self-defined scientific area

5. Protect students, fellows and postdoctoral fellows involved in collaborative projects from exploitation 6. Ensure that effective mechanisms exist to eliminate, control or manage conflicts of interest in the partnership

7. Ensure that all investigators and both partners retain their proprietary and intellectual property rights throughout and after the partnership

8. Ensure that data-sharing arrangements are explicit and that all rights to access

data are fairly negotiated at the outset of the partnership

9. Establish parameters for what type of projects will and will not be funded (e.g., add-on projects, training, pilot studies)

10. Create ways to protect each party

from an unexpected end to the partnership

11. Formally assess the efficiency, effectiveness and achievements of the partnership on an annual basis

12. Ensure that clear, comprehensive and efficient procedures exist for all governance entities of the partnership and are known to all investigators 13. Ensure that all investigators, staff and other participants in the partnership have adequate training in the responsible conduct of research and related ethical issues

14. Ensure that all projects in the partnership aim to satisfy the highest ethical standards

15. Ensure the right of all researchers associated with the partnership to publish 16. Disseminate all research results at the conclusion of collaborative studies in a timely fashion

17. Ensure that authorship follows ICMJE guidelines

18. Maintain competitive advantage in the specified research domains 19. Structure the research to maximize potential benefit for communities and society

20. Structure the partnership to have the best chance of benefiting both partners and harming neither

21. Widely publicize the partnership agreement and collaborative opportunities to the public and employees

22 . Establish procedures for frequent and effective communication between partners

23. Ensure that both partners are aware of other partnerships each may be involved in include a wider range of Institute and university investigators in the invitation to propose studies; 4) publicize the collaboration's distinctive conflict of interest policies; and 5) proactively assess investigators' concerns about the collaboration and provide investigators with more opportunities to learn about the collaboration and provide input.

As a result of the IUBC evaluation and the more than two decades of research collaboration with industry, the Regenstrief Institute has launched an Industry Research Office (IndRO) that facilitates conversations with prospective industry funders, identifies Regenstrief and other university principle investigators and co-investigators, helps design protocols and write proposals, manages communication and contracts, and follows the principles and benchmarks for a wider range of investigators, funders, and studies. The overriding goal of the IndRO is to provide academic researchers with alternative sources of funding their research as federal sources become increasingly constrained. In addition to faculty and staff salaries for performing research, funds from industry-sponsored studies support IndRO's management infrastructure, local clinical data repositories, and other research resources. To maintain the studies' intellectual independence and scholarly focus, all industry contracts contain a clause giving the investigators the right to publish any and all study results, and an article submitted to a peer-reviewed journal is the final required deliverable of all contracts.

The moral outrage engendered by past misbehavior on the part of the drug and device industry and academic researchers can affect all financial relationships between medical schools and industry. ${ }^{32,33}$ If stringent ethical guidelines are followed, private sector companies can be an important source of funding for ethical, high-quality, important academic research. Universities must develop and implement policies and procedures to maximize the effectiveness and ethical conduct of all research, regardless of funding source. We are confident that this is possible and that industry, academic medical scientists, and the patients and communities they serve can all benefit.

Conflict of Interest: Dr. Tierney has received research funding from Merck Sharp \& Dohme, Eli Lilly, Bristol-Myers Squibb, GlaxoSmithKline, Caremark, and Integrated Disease Management, Inc. He has never owned stock in individual medically related companies and has never received honoraria, speaking fees, or personal income from any medically related company. He is the President and CEO of the Regenstrief Institute, Inc., which has an Industry Research Office that facilitates research contracting between academic investigators and the private sector. All residual funds realized by this research support Regenstrief's local research infrastructure. No funds result in bonuses or additional income to investigators or staff.

Dr. Meslin does not now but has previously received consulting fees from Eli Lilly. He sits on the Science and Industry Advisory Committee of Genome Canada, for which he receives an annual honorarium. Dr. Kroenke has received research funding from Eli Lilly, Pfizer, and Wyeth. He does not now but has previously received consulting fees and/or honoraria from Eli Lilly, Wyeth, Forest Laboratories, and BristolMyers Squibb. He has no investments in individual for-profit companies. 
Corresponding Author: William M. Tierney, MD; Regenstrief Institute, Inc., 410 West Tenth Street Suite HS200O, Indianapolis, IN 46202, USA (e-mail: wtierney@regenstrief.org).

\section{REFERENCES}

1. Moses H III, Matheson DHM, Cairns-Smith S, George BP, Palisch C, Dorsey R. The anatomy of medical research: US and international comparisons. JAMA. 2015;313:174-89.

2. National Center for Advancing Translational Sciences. Discovering new therapeutic uses for existing molecules. Available at: http://www.ncats. nih.gov/research/reengineering/rescue-repurpose/therapeutic-uses/ therapeutic-uses.html. Accessed April 25, 2015.

3. National Institutes of Health. Accelerating medicines partnerships. Available at: http://www.nih.gov/science/amp/index.htm. Accessed April 25, 2015.

4. National Institutes of Health. NIH launches collaborative program with industry and researchers to spur therapeutic development. Available at: http://www.nih.gov/news/health/may2012/od-03.htm. Accessed April 25, 2015.

5. Patent and Trademark Law Amendments Act of 1980, Pub. L. No. 96-517, 94 Stat. 3015, 69331 (1980) (codified as amended at 35 U.S.C. ch. 30 (2011)).

6. Patient Protection and Affordable Care Act, Pub. L. No. 111-148, 124 Stat. 119 (2010); Sec. 6002: Transparency Reports and Reporting of Physician Ownership or Investment Interests (codified as 42 U.S.C.A. § 1320a-7h).

7. USInflation.org. US inflation rate calculator. Available at: http:// usinflation.org/us-inflation-rate-calculator/. Accessed July 7, 2015.

8. Singh S, Loke YK. Drug safety assessment in clinical trials: methodological challenges and opportunities. Trials. 2012;13:138.

9. Farwell WR, Stump TE, Wang J, Tafesse E, L'Italien G, Tierney WM. Weight gain and new onset diabetes associated with olanzapine and risperidone. J Gen Intern Med. 2004; 19:1200-5.

10. Psaty BM, Meslin EM, Breckenridge A. A lifecycle approach to the evaluation of FDA approval methods and actions: opportunities provided by a new IOM report. JAMA. 2012;307(23):2491-2.

11. Jain SH, Rosenblatt M, Duke J. Is big data the new frontier for academicindustry collaboration? JAMA. 2014;311:2171-2.

12. Melander H, Ahlqvist-Rastad J, Meijer G, Beermann B. Evidence b(i)ased medicine-selective reporting from studies sponsored by pharmaceutical industry: review of studies in new drug applications. BMJ. 2003;326:1171-3.

13. Godlee F, Smith J, Marcovitch $\mathbf{H}$. Wakefield's article linking MR vaccine and autism was fraudulent. BMJ. 2011;342:c7452.
14. Cyranoski D. Research integrity: cell-induced stress. Nature. 2014;511(7508):140-3.

15. Hollon T. Researchers and regulators reflect on first gene therapy death. Nat Med. 2000;6:6.

16. Olsen AK, Whalen MD. Public perceptions of the pharmaceutical industry and drug safety: implications for the pharmacovigilance professional and the culture of safety. Drug Saf. 2009;32:805-10.

17. Kroenke K, Spitzer RL, Williams JBW, Löwe B. The Patient Health Questionnaire somatic, anxiety, and depressive symptom scales: a systematic review. Gen Hosp Psychiatry. 2010;32:345-59.

18. Kroenke K, Spitzer RL, Williams JBW. The PHQ-9: validity of a brief depression severity measure. J Gen Intern Med. 2001;16:606-13.

19. Web of Science. Available at: www.isiknowledge.com. Accessed April 25, 2015.

20. McHorney CA, Ware JE Jr, Raczek AE. The MOS 36-Item Short-Form Health Survey (SF-36): II. Psychometric and clinical tests of validity in measuring physical and mental health constructs. Med Care. 1993;31:247-63.

21. Newman JC, Feldman R. Copyright and open access at the bedside. N Engl J Med. 2011;365(26):2447-9.

22. Song F, Parekh S, Hooper L, et al. Dissemination and publication of research findings: an updated review of related biases. Health Technol Assess 2010;14:iii. ix-xi, 1-193.

23. Bero L. Industry sponsorship and research outcome: a Cochrane review. JAMA Intern Med. 2013;173:580-1.

24. Rennie D. Thyroid storm. JAMA. 1997;277:1238-43.

25. Blumenthal D, Campbell EG, Anderson MS, Causino N, Louis KS. Withholding research results by academic life scientists: evidence from a national survey of faculty. JAMA. 1997;277:1224-8.

26. Tierney WM, Gerrity MS. Scientific discourse, corporate ghostwriting, journal policy, and public trust. J Gen Intern Med. 2005;20:550-1.

27. Kroenke K, West SL, Swindle R, et al. Similar effectiveness of paroxetine, fluoxetine, and sertraline in primary care: a randomized trial. JAMA. 2001;286:2947-55.

28. Krimsky S. Do financial conflicts of interest bias research? An inquiry into the "funding effect" hypothesis. Sci Technol Hum Values. 2013;38:566-87.

29. Sterne JA. Why the Cochrane risk of bias tool should not include funding source as a standard item. Cochrane Database Syst Rev. 2013; 12:ED000076

30. Carpenter WT Jr, Koenig JI, Bilbe G, Bischoff S. At issue: a model for academic/industry collaboration. Schizophr Bull. 2004;30:997-1004.

31. Meslin EM, Gaffney MM, Guaid KA, Schwartz PH, Pitt AR, Rager JB. Final summary report: Review of the Merck-Regenstrief partnership. Available at: http://hdl.handle.net/1805/6044. Accessed July 7, 2015.

32. Rosenbaum L. Beyond moral outrage - Weighing trade-offs of COI regulation. N Engl J Med. 2015;372:2064-8.

33. Steinbrook R, Kassirer JP, Angell M. Justifying conflicts of interest in medical journals: a very bad idea. BMJ. 2015;350:h2942. 